\title{
LA USCO IMPLEMENTA RECOMENDACIONES DE LA MISIÓN CIENCIA, EDUCACIÓN Y DESARROLLO Y POLITICA NACIONAL DE CIENCIA, Y TECNOLOGIA
}

Por: HILDA MARINA BOHORQUEZ LOZANO Profesora: Universidad Surcolombiana, Matemáticas y Física (Neiva) Coordinadora de Práctica - Programa de Matemáticas y Física Coordinadora del Proyecto CIPCIT

A mediados del año 1994 la MISIÓN CIENCIA, EDUCACIÓN Y DESARROLLO presentó al gobieno, el documento "COLOMBIA: AL FILO DE LA OPORTUNIDAD" en el que hace importantes análisis a la Ciencia, la Educación y el Desarrollo en nuestro pais. En la parte final del documento se presentan un conjunto de recomendaciones, de las cuales es importante resaltar las que tienen que ver con la ENDOGENIZACIÓN DE LA CIENCIA Y LA TECNOLOGIA; lo cual significa que éstas deben pasar a formar parte propia de cada individuo, a tal punto que se familiarize con ellas como con cualquier situación que hace parte de su cotidianeidad y la de APROPIACIÓN SOCIAL DE LA CIENCIA, lo que implica que ésta pase a formar parte de la sociedad como lo es el folclor, las costumbres regionales, etc.

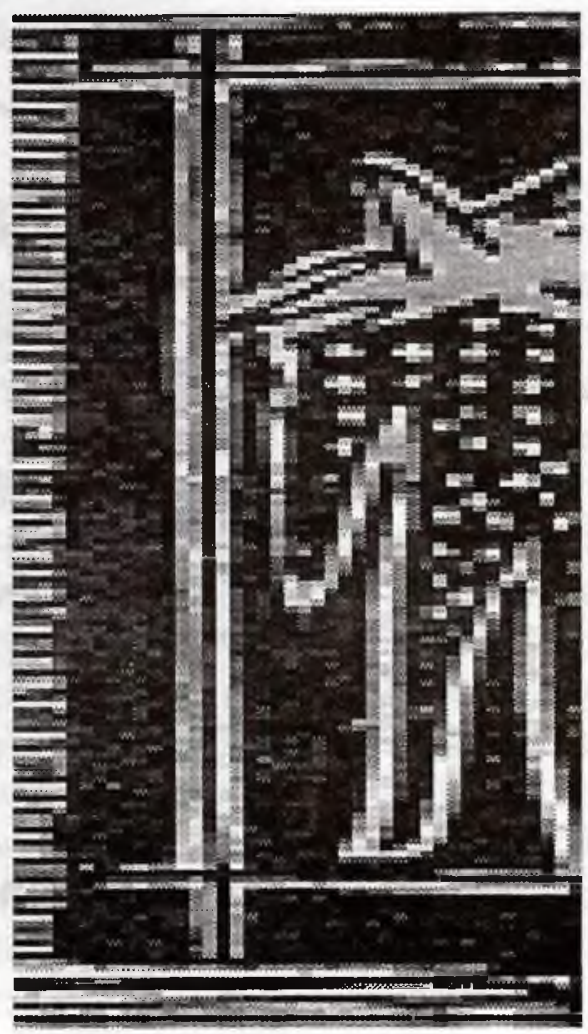


Por otra parte el CONSEJO NACIONAL DE POLITICA ECONOMICA Y SOCIAL (CONPES) en noviembre 1994 recoge tales recomendaciones planteando en su documento relativo a la política nacional de ciencia y tecnología como objetivo general "INTEGRAR LA CIENCIA Y LA TECNOLOGIA A LOS DIVERSOS SECTORES DE LA VIDA NACIONAL, BUSCANDO INCREMENTAR LA COMPETITIVIDAD DEL SECTOR PRODUCTIVO EN EL CONTEXTO DE UNA POLITICA DE INTERNACIONALIZACION DE LA ECONOMIA , Y MEJORAR LA CALIDAD DE VIDA DE LA POBLACIÓN COLOMBIANA"

Para lograr dicho objetivo plantea cinco estrategias, de las cuales se resalta la 5, que dice 'INTEGRAR LA CIENCIA Y LA TECNOLOGIA A LA SOCIEDAD Y A LA CULTURA COLOMBIANAS A TRAVES DE UN PROGRAMA DE ENSEÑANZA, DIVULGACIÓN Y POPULARIZACIÓN DE LAS MISMAS". La implementación de esta estrategia requiere de acciones a ser asumidas por diferentes entidades y apoyadas financierarnente por otras.

COLCIENCIAS, entidad que financia proyectos de investigación en pro del desarrollo de nuestro país, ha establecido para el alcance de lo anterior 11 líneas de las cuales se recoge aquí la correspondiente a la ENSEÑANZA Y POPULARIZACIÓN DE LAS CIENCIAS la cual destaca como principio eje de la misma que,
Ciencia que no se divulge no es Ciencia.

Por su parte, la USCO y la GOBERNACION DEPARTAMENTAL, en un convenio especial de trabajo liderador del desarrollo regional del Huila en cuanto a Ciencia y Tecnología, elaboraron EI PLAN DE ACCIÓN DEPARTAMENTAL EN CIENCIA Y TECNOLOGIA para el Huila teniendo en cuenta los documentos anteriores y el resultado de una investigación diagnostica, en el cual incluyeron el programa de Investigación y Desarrollo Institucional. Dentro de este programa ubicaron el proyecto "ESTRUCTURACIÓN DE UN CENTRO DE INTERACCIÓN DEL PUBLICO CON LA CIENCIA, LA INGENIERIA Y LA TECNOLOGIA", -C.I.P.C.I.T.". Paralelamente este centro fue instituciolizado en el mes de Agosto por el Consejo Superior Universitario de la Universidad Surcolombiana (USCO), dentro del espíritu de contribuir a lograr el objetivo y en especial la estrategia de Popularización de la Ciencia y la Tecnología, de la Política Nacional descrita. Es importante resaltar que esta propuesta habia sido avalada y apoyada en su etapa de formulación, por parte de la Asociación Colombiana para el Avance de la Ciencia. Se aspira a que el CIPCIT haga parte de Ia RED NACIONAL DE MUSEOS O CENTROS INTERACTIVOS DE CIENCIA Y TECNOLOGIA, para 10 cual participó en Convocatoria Nacional hecha por la Universidad Nacional de Colombia y COLCIENCIA. Sabremos los resultados a finales de marzo del presente año. 


\section{LA PROPUESTA}

Consiste en estructurar, construir y dotar un CENTRO (lugar) destinado a la INTERACCIÓN (Acción mutua) del PUBLICO (Niños, jóvenes, adultos) que por iniciativa personal desean divertirse con la CIENCIA, LA INGENIERIA Y LA TECNOLOGIA, a través de:

-MODELOS Y MONTAJES DIDÁCTICOS INTERACTIVOS, elaborados por profesores y estudiantes de la USCO (Facultades de Salud, Educación e Ingeniería), y de otras Universidades, por estudiantes de establecimientos educativos de Básica Primaria, Secundaria y Media, por profesionales de Centros de desarrollo Industrial de la región y por particulares interesados en hacerlos.

-ACTIVIDADES ACADÉMICAS PERIÓDICAS, como: Talleres, cursos,seminarios, conferencias, etc., desarrollados en el CIPCIT, en colegios y en otros centros.

-ACTIVIDADES DE DIVULGACIÓN, consistentes en producción de material audiovisual como: videos, películas y software educativo.

- CENTRO DE DOCUMENTACIÓN, para el acceso rápido a la información, consulta bibliográfica red Internet, divulgación de trabajos que relacionen personas interinstitucionalmente.

- INTERCAMBIO PERIÓDICO CON ENTIDADES SIMILARES, tanto de experiencias y materiales producidos por ellas.

- PROMOCIÓNY REALIZACIÓN DE ACTIVIDADES CIENTÍFICAS JUVENILES, como: muestras intraescolares e interinstitucionales, congresos, olimpiadas, clubes, excursiones y campamentos.

- CORREO PERIÓDICO ALREDEDOR DE LA CIENCIA, entre científicos y jóvenes.

- CAMPAMENTOS EN EL CENTRO: para ser realizados alrededor de temas de la ciencia y la tecnología con juegos, fogatas, talleres, etc.

Todas estas actividades girarán en los campos de: LAS CIENCIAS FÍSICAS, LA ECOLOGÍA Y EL MEDIO AMBIENTE, LAS MATEMÁTICAS YLOS SISTEMAS, LABIOLOGÍA Y EL INGENIO HUMANO EN LA CIENCIA Y LA TECNOLOGÍA.

El desarrollo de las actividades mencionadas se orienta en torno a una pauta principal: LA INTERACCIÓN, entendida ésta como una acción mutua entre el PÚBLICO y la CIENCIA, LA INGENIERÍA Y LA TECNOLOGÍA. 


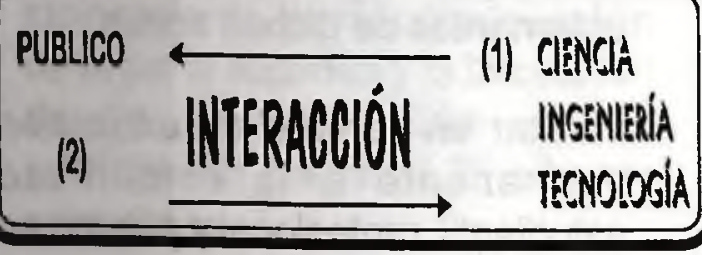

La acción (1) de La Ciencia. La Ingeniería y La Tecnología sobre el Público, buscará crear una ruptura de un esquema aprendido desde la infancia, en el hogar y en la escuela tradicional, marcado por expresiones como: No cojas!, No toques!, No molestes!, No preguntes!, etc., que limitan la naturaleza espontánea del ser humano, y que se manifiesta en la exploración del mundo que le rodea a través de sus sentidos: el gusto, el olfato, el oído, la vista y el tacto.

Esta acción se observa en la medida en que las personas manifiesten una actitud positiva tendiente a encontrar y comprender capacidades humanas en sí mismas en los visitantes y en las personas que trabajan con Ciencia y Tecnología; a generar mejores actitudes y aptitudes bacia éstas y a orientar mejor sus capacidades naturales y espontáneas como seres humanos de SER, CONOCER Y VIVIR.

En síntesis la acción de la Ciencia, la Ingeniería y la Tecnología sobre las personas deberá potenciar SU DESARROLLO HUMANO $Y$ ORGANIZACIÓNAL DURADEROS Y DINÁMICOS, FAVORECIENDO CON ELLO ELDESARROLLO DEL MEDIO EN EL QUE VIVEN: EL DEPARTAMENTO DEL HUILA Y LAS REGIÓNES CIRCUNVECINAS.
La acción (2) del Público sobre la Ciencia, la Ingeniería y la Tecnología, más a largo plazo, se traducirá en el siguiente hecho:

CUANDO LOS SERES HUMANOS (Para el caso los huilenses) ENTREN EN UN AUTENTICO DESARROLLO HUMANO, CONSTANTEMENTE ESTARÁN DESARROLLANDO VALORES, APRENDIENDO CONTINUA Y GUSTOSAMENTE, NO PARA ESCALAR SINO PARA HACER MEJOR LO QUE HACEN Y ESTARÁN ASÍ EN CAPACIDAD DE APORTAR IDEAS PARA EL DESARROLLO DE LA CIENCIA, LA INGENIERÍA Y LA TECNOLOGÍA EN BIEN DEL ENTORNO QUE LES RODEA.: EL DEPARTAMENTO.

De acuerdo a la pauta descrita, se pretende con el desarrollo de la propuesta:

- Complementar la acción del sistema educativo iormal.

- Dar oportunidad a los estudiantes de los diferentes niveles del sistema educativo para desarrollar su creatividad en condiciones que el mismo sistema no puede ofrecerle (Interés personal tiempo disponible, etc.).

- Provocar la participación al menos como espectadores de la familia de los participantes y de la comunidad en general promoviendo con ello una incidencia aunque indirecta de medio multiplicador.

- Constituir las actividades del Centro en alicientes que permitan mejorar la actitud y aptitud científicas de los estudiantes participantes y 


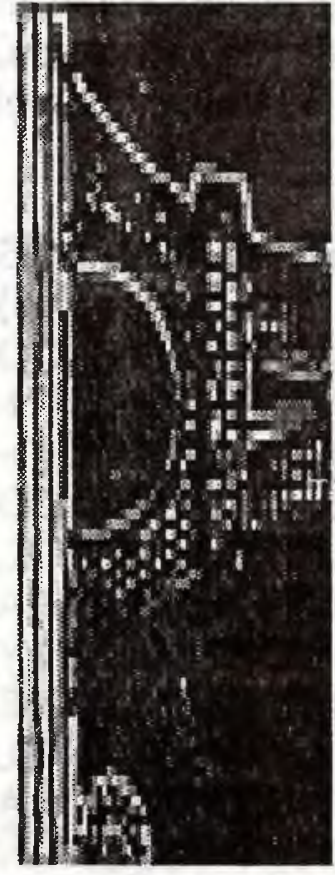

vis it antes permitiéndoles sentir la ciencia como elemento más propio y cercano a su cotidianeidad.

Establecer entre los estudiantes participantes, un auténtico espíritu de trabajo en squipo.

- Incentivar en los estudiantes que inician trabajos para el CIPCIT, la disciplina investigativa, como preámbulo a investigaciones de nivel superior.

- Contribuir y elevar el nivel de educación científica en los educandos y educadores del Departamento, a través de las actividades académicas implementadas por el CIPCIT

- Fomentar y/o crear redes de intercambio de exposiciones de interés con otras universidades, y centros similares y con empresas a nivel regional y nacional.

- Fomentar periódicamente exposiciones y muestras especiales que permitan una mayor dinámica cultural y científica en la región Surcolombiana

- Promover la extensión de los servicios del CIPCIT a diferentes regiones del departamento en coordinación con alcaldías y estamentos de dichas zonas.

- Prestar un servicio de exhibición permanente a la comunidad estudiantil, profesional y público en general.

- Contribuir al desarrollo de las estrategias y acciones planteadas en la Política Nacional actual de Ciencia y Tecnología.

Se ha iniciado el desarrollo de la propuesta con el apoyo institucional municipal, departamental y se espera obtener financiación en otras entidades como COLCIENCIAS ICFES, CORPES, ECOPETROL, etc.

Para la primera etapa de su desarrollo, dos años, se tienen previstas:

- Realización de actividades básicas para mercadeo

- Promoción y realización de actividades académicas periódicas relativas a Ciencia, Ingeniería y Tecnología.

- Realización de MUESTRAS CIENCIA, TECNOLOGICAS Y CREATIVIDAD

- Elaboración de modelos didácticos interactivos por parte de estudiantes

- Actividades de apoyo interinstitucional conducentes a perfeccionar y estructurar aspectos relativos a funcionamiento del CIPCIT 
- Trabajo de diseño y perfeccionamiento para construcción de la parte locativa y la iniciación de la construcción de la edificación hacia el $2^{\circ}$ año de esta etapa.

En la segunda etapa, prevista para otros dos años, se desarrollarán las siguientes acciones:

- Finalizar la construcción del CIPCIT y dotar el Centro según lo previsto y lo resultante del perfeccionamiento de la propuesta.

- Montaje de exposiciones núcleo diseñadas.

- Continuidad de las actividades iniciales que gestaron o dieron vida al Centro.

La tercera etapa será la consolidación de Centro con las actividades sobre las cuales serán el eje y vida del Centro, y con las cuales inició. En esta etapa el Centro deberá tener una estructura administrativa con su respectiva planta de personal.
Desde cualquier punto de la geografía Colombiana, todo ciudadano puede vincularse al desarrollo de esta propuesta. Inicialmente se ha planteado la Invitación a quienes deseen aportar, para que valoren la propuesta desde sus sanos y valiosos criterios y aporten ideas y sugerencias para un mejor desarrollo de ésta. Pueden hacerlas llegar al Programa de Matemáticas y Física, 0 a la Decanatura de la Facultad de Educación de la Universidad Surcolombiana.

Como la base de la propuesta es la INTERACCIÓN, desde ya, sus acciones en cuanto a la misma, en el sentido de aportar ideas, SERÁN DE MUCHA IMPORTANCIA.

En 1996 se inició el desarrollo de algunas de las actividades previstas, y continuarán para los años subsiguientes. Podrán los interesados asistir a ellas y si desean ser participantes activos, también será posible. Pueden serlo, diseñando montajes de modelos ilustrativos de algún aparato ó de un fenómeno natural en especial que les haya inquietado

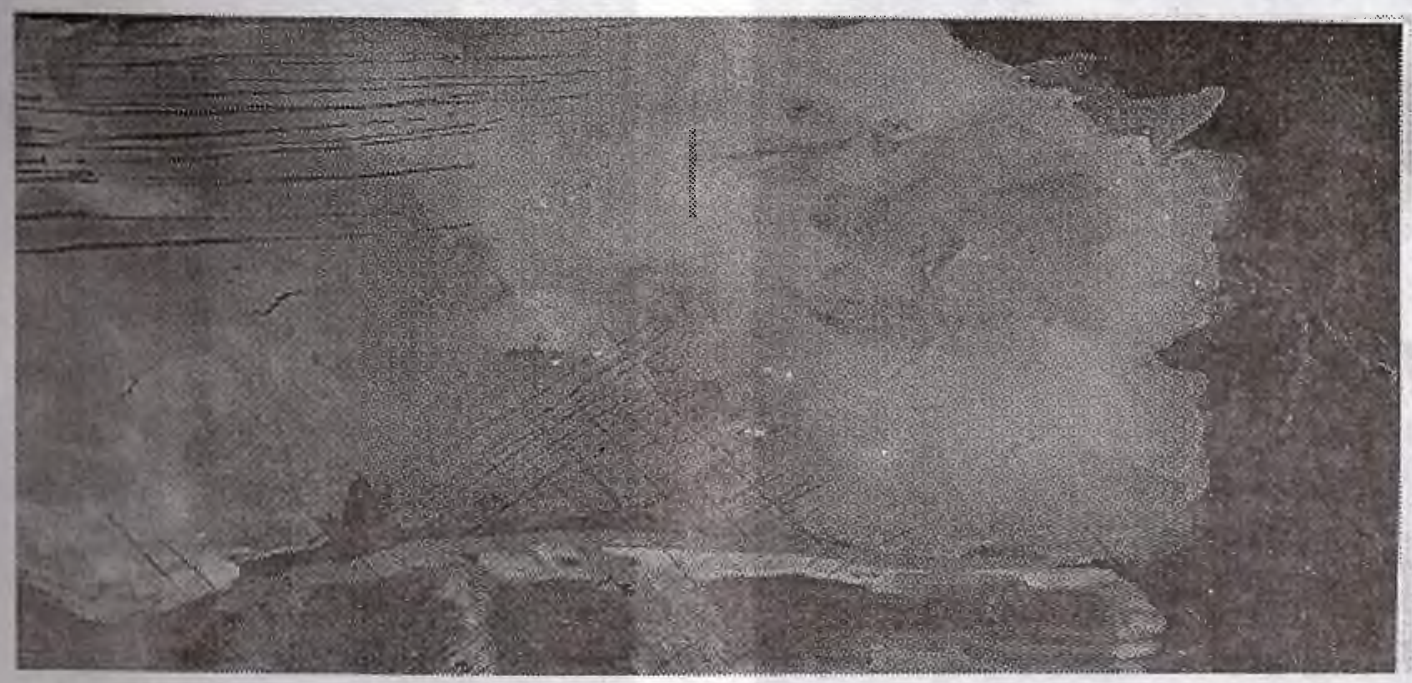


y sobre el que hayan hecho estudio ó deseen hacerlo, ó sobre un experimento en especial. Estos podrán ser presentados en MUESTRAS DE CIENCIA, TECNOLOGÍA Y CREA-TIVIDAD, organizadas por el CIPCIT. NO SERÁN INVENTOS NI COSAS SOFISTICADAS. Serán montajes sencillos destinados a retroalimentar conocimientos de la ciencia en los campos anotados. Es posible que resulten muchos tan interesantes que valga la pena perfeccionarlos para que luego puedan pasar a hacer parte de la colección de montajes del CIPCIT, tarea que podrá realizar el autor inicial $u$ otro que resulte intere-sado en hacerlo responsablemente.

Lo importante para iniciar, es la existencia del deseo real de hacer el trabajo mejor con los fines divulgativos para los cuales siempre serán elaborados. Para continuar, la disciplina y entereza de querer resolver una inquietud personal o de

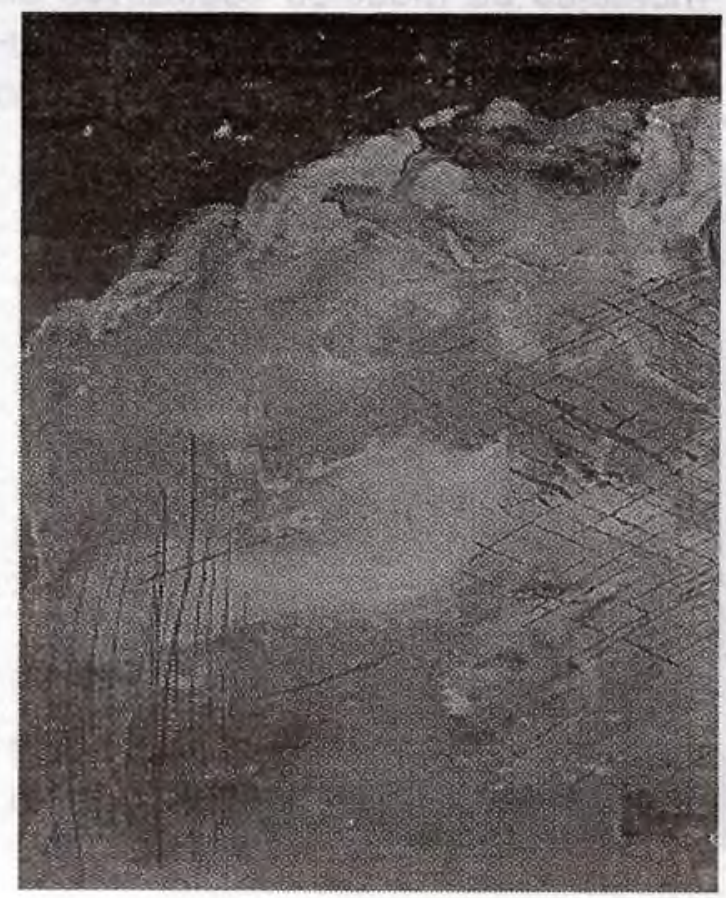

aportar para resolver la de otros, lo que originará otras inquietudes nuevas. De esta manera se estará generando el espíritu de investigación en la población huilense, y con ello estaremos aportando al desarrollo de nuestra región.

Es importante finalizar este artículo resaltando un párrafo de un escrito de MICHEL NOVAK, el cual se puede tomar como una de las razones y a su vez un eje orientador del CIPCIT. La riqueza de los pueblos no se encuentra en sus recursos naturales. De ser así Brasil sería enormemente rico y Japón tremen-damente pobre. El origen verdadero de la riqueza de las naciones está en el capital humano. En la creatividad de sus ciudadanos. Aquellos países que han construído los sistemas políticos que mayormente liberan y respaldan la creatividad de sus ciudadanos, se enriquecen rápidamente aún sin tener recursos naturales." \&

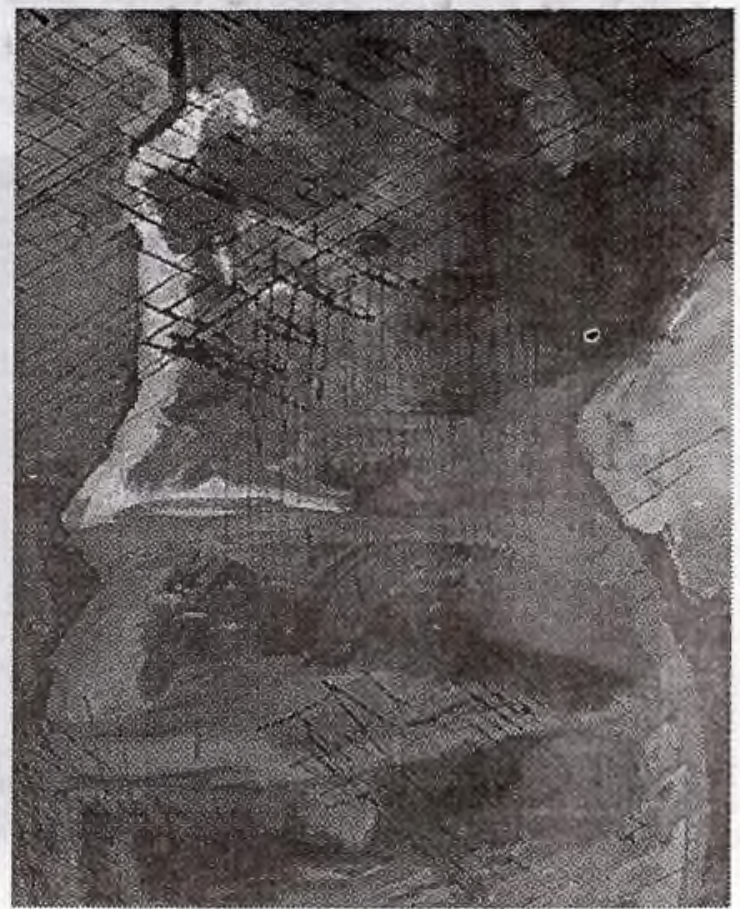




\section{BIBLIOGRAFIA}

- ASOCIACION COLOMBIANA PARA EL AVANCE DE LAS CIENCIAS (A.C.A.C.), Centro de Ciencia y Tecnología. Estudio de Prefactibilidad (Santafé de Bogotá) 1.992.

- BOHÓRQUEZ L., HILDA M. Propuesta para la Estructuración, construcción y Dotación de un Centro de Interacción del Público con la Ciencia y Te, la Ingeniería y la Tecnología. (Neiva), 1.993.

- BUSCAGLIA, LEO. Amar, Vivir y Aprender. Edlt. Diana. 1985.

- COLCIENCIAS. HACIA UN DESARROLLO SOSTENIBLE Y EQUITATIVO. Santafé de Bogotá. Marzo, 1995.

- CONPES. POLÍTICA NACIÓNAL DE CIENCIA Y TECNOLOGÍA. Santafé de Bogotá. Noviembre, 1994.

- MISIÓN DE CIENCIA, EDUCACIÓNY DESARROLLO. "r.OLOMBIA: AL FILO DE LA OPOR-TUNIDAD”. Santafé de Bogotá. Junio de 1994.

- RODERICK, O'CONNOR. Memorias de Seminario para Líderes del desarrollo regional Neiva, Julio 1.995. $8 \%$ 